\title{
Management of Intracoronary Thrombus
}

\author{
Janarthanan Sathananthan, Timothy J. Watson, \\ Dale Murdoch, Christopher Overgaard, Deborah Lee, \\ Deanna Khoo, and Paul J. L. Ong
}

\subsection{Introduction}

Partial or complete occlusion of the infarct-related artery (IRA) with intracoronary thrombus (ICT) is the pathognomonic hallmark of patients presenting with ST-elevation myocardial infarction (STEMI). Thrombus burden can be highly variable, but its presence is associated with worse outcomes, including lower procedural success, increased abrupt vessel closure and an increased frequency of major inhospital complications including death and recurrent myocardial infarction (MI). ICT poses a unique series of challenges, but appropriate management is an essential prerequisite for successful primary percutaneous coronary intervention (PPCI). This can largely be achieved using a combination of pharmacological and mechanical approaches prior to coronary stent insertion.

In this chapter, we discuss the causes, sequelae and treatment of intracoronary thrombus specific to patients presenting with STEMI.

\section{J. Sathananthan $(\bowtie)$}

St. Pauls Hospital, Vancouver, BC, Canada

T. J. Watson

HSC Medical Center, Menara HSC, Kuala Lumpur, Malaysia

D. Murdoch

Department of Cardiology, The Prince Charles Hospital, Brisbane, QLD, Australia

The University of Queensland, St. Lucia, QLD, Australia

C. Overgaard

Cardiac Catheterization Laboratories and Coronary Intensive Care Unit,

Toronto General Hospital, University Health Network, Toronto, ON, Canada

D. Lee $\cdot$ D. Khoo $\cdot$ P. J. L. Ong

Tan Tock Seng Hospital, National Healthcare Group, Singapore 


\subsection{Pathophysiology of Intracoronary Thrombus Formation}

In the majority of cases, the nidus for thrombogenesis is erosion of an underlying atherosclerotic plaque and subsequent exposure of thrombogenic subendothelial matrix and plaque to circulating platelets [1]. Plaque rupture initiates the coagulation cascade in one of two distinct but ultimately synergistic pathways (Fig. 10.1). In the first pathway, platelet glycoprotein VI binds directly with collagen exposed by the denuded endothelium. Concurrently platelet glycoprotein Ib-V-IX interacts with collagen-bound von Willebrand factor ( $\mathrm{vWf}$ ). This process triggers platelet activation, adherence and accumulation to the vessel wall leading to formation of 'white' thrombi. In contrast, the second pathway leads to formation of 'red' thrombi where tissue factor initiates a proteolytic cascade which leads to generation of thrombin which in turn converts fibrinogen to fibrin while also triggering activation and accumulation of platelets through release of various agonists including adenosine, thromboxane $\mathrm{A}_{2}$ and serotonin. These agents activate other platelets, thereby amplifying the thrombogenic process. Coronary thrombus consists of platelets, erythrocytes, inflammatory cells and fibrin. The thrombin-generating process leads to denser, more fibrin-rich thrombus which becomes progressively more difficult to disrupt (both pharmacologically and mechanically) with time [2-5].

1. Platelet adhesion

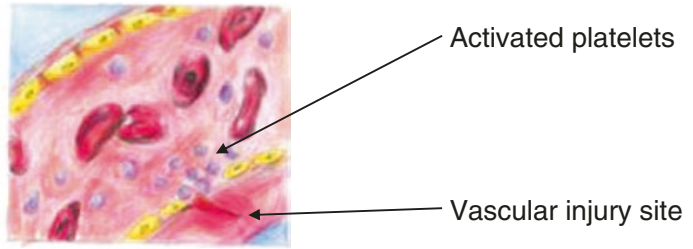

2. Platelet release reactions

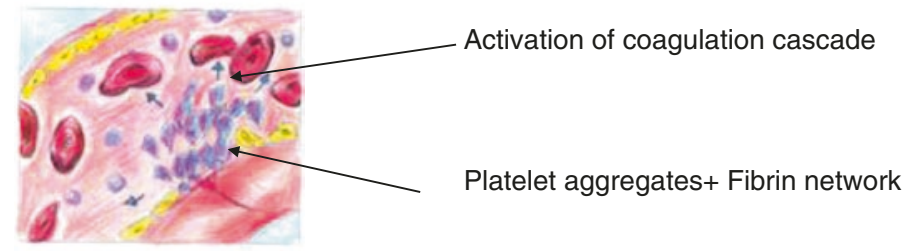

3. Platelet aggregation \& clot formation

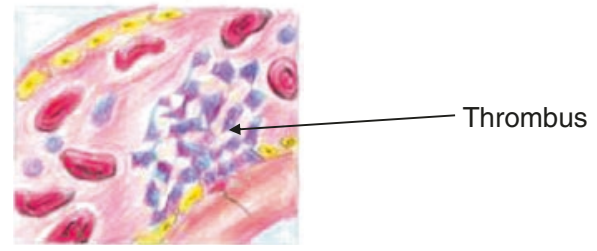

Fig. 10.1 Intracoronary effects of coagulation cascade. [Courtesy of Miss Christina YS Wong] 
The more friable the thrombus burden is at the time of PCI, the more susceptible it is to mechanical intervention. However, particularly with late presentations, thrombus can also be resistant to coronary devices, including balloons and aspiration catheters. Thrombus variability relates to the two types of fibrin fibres that are deposited at the time of thrombogenesis, including thin dense fibres (which are poorly dissolved by thrombolytic agents and mechanical devices) and thick fibrin fibres (which are more susceptible to treatment).

\subsubsection{Grading Coronary Thrombus}

Grading tools are useful to quantify the thrombus burden in patients presenting with STEMI. The TIMI (thrombolysis in myocardial infarction) thrombus grading scale (Fig. 10.2) is commonly used and is a simple numerical scale ranging from grade 0 (no thrombus) to grade 5 (very large thrombus that completely occludes vessel flow) $[6,7]$. The scale is subject to variability in interpretation and can be simplified to a binary system: low-grade thrombus (TIMI 1-3) and high-grade thrombus (TIMI 4-5) [8]. Visual grading tools also tend to underestimate thrombus size compared to other tools such as intracoronary imaging.

\section{TIMI Thrombus Grade}

0 . No cine-angiographic characteristics of thrombus present

1. Possible thrombus present. Angiography shows characteristics such as reduced contrast density, haziness, irregular lesion contour, or a smooth convex meniscus at the site of total occlusion suggestive but not diagnostic of thrombus

2. Thrombus present, small size: definite thrombus with greatest dimensions less than or equal to half vessel diameter

3. Thrombus present, moderate size: definite thrombus but with greatest linear dimension greater than half but less than 2 vessel diameters

4. Thrombus present, large size: as in grade 3 but with the largest dimension greater than or equal to 2 vessel diameters

Fig. 10.2 TIMI thrombus

5. Total occlusion 


\subsubsection{Intracoronary Thrombus and Clinical Outcomes}

The presence of ICT during acute coronary syndromes often results in suboptimal coronary reperfusion and worse clinical outcomes $[7,9,10]$. The effect of thrombus on the microvascular circulation can be quantified using degree of ST-segment resolution or the myocardial blush grade [11]. The myocardial blush grade is used as a marker of a perfusion in the capillary level at the tissue level. Both the degree of ST-segment resolution and myocardial blush grade have been shown to be independent markers of mortality [12].

Use of a grading tool allows a consistent method for thrombus assessment and correlation to clinical outcomes. Importantly they can also aid in management decisions prior to and during PCI. The presence of ICT and distal embolization to the microcirculation can lead to persistent chest pain, electrocardiogram changes and distal vessel occlusion. Even if normal epicardial flow is achieved, distal embolization and microvascular obstruction are associated with increased infarct size, reduced ventricular function and worse survival. Due to the negative clinical consequences of incomplete thrombus resolution, it is essential that interventional cardiologists consider strategies to prevent and treat ICT during STEMI.

\subsection{Management}

The extent of ICT observed during PPCI correlates strongly with both procedural success and clinical outcome. Due to the complex nature of intracoronary thrombogenesis, a multi-faceted and systematic approach is required to achieve successful thrombus dissolution. This includes arrest of the coagulation cascade (usually achieved using pharmacotherapy), flow restoration (usually through mechanical techniques) and occasionally with the use of thrombus extraction tools. Thrombus extraction may theoretically limit distal embolization of thrombus, thereby preventing microvascular obstruction and incomplete microvascular reperfusion.

\subsubsection{Pharmacological Interventions}

Comprehensive review of pharmacological interventions utilized during PPCI is available in Chaps. 8 and 9. Due to the self-propagating nature of thrombogenesis, early and effective pharmacological interventions to inhibit this process are key. At point of initial STEMI diagnosis, patients should receive a loading dose of aspirin (e.g. $300 \mathrm{mg}$ or institutional practice) and a $\mathrm{P}_{2} \mathrm{Y}_{12}$ antagonist (e.g. clopidogrel $600 \mathrm{mg}$, prasugrel $60 \mathrm{mg}$ or ticagrelor $180 \mathrm{mg}$ ) [13-15]. In some healthcare systems, community STEMI diagnosis may occur, and early administration of these oral antiplatelet agents may be feasible [16]. More commonly, administration occurs in the emergency department; with shorter transfer times to the cardiac catheterization laboratory, this could lead to suboptimal platelet inhibition during the PPCI procedure [17]. Such considerations are important, particularly for the immediate 
peri-PCI period in a freshly stented patient who is naïve to antiplatelet therapy and in cases where thrombus burden is significant. This has led to development of rapidonset intravenous $\mathrm{P}_{2} \mathrm{Y}_{12}$ antagonists (e.g. cangrelor), which may prove useful as a bridge until the maximal antiplatelet effect of oral agents is realized [18]. An intravenous $\mathrm{P}_{2} \mathrm{Y}_{12}$ agent may also be advantageous in STEMI patients that are intubated or unable to have oral agents. However, these agents are not broadly available.

Peri-procedural anticoagulation is generally achieved using unfractionated heparin or in some catheter laboratories using low-molecular weight heparin (e.g. enoxaparin), factor Xa inhibition (e.g. fondaparinux) or a reversible direct thrombin inhibitor (bivalirudin) $[19,20]$. Each strategy has individual merit and choice is therefore largely determined by operator/unit preference or cost. The progressive shift from transfemoral to transradial intervention amongst patients with STEMI has vastly reduced vascular access complications such as bleeding and improved procedural safety [21, 22]. Consequently, the purported advantage of agents such as bivalirudin that reduce bleeding to improve clinical outcomes may be less marked [23].

In our opinion, peri-procedural anticoagulation is best achieved in keeping with the standard protocol each laboratory uses for PCI in stable patients without need for modified protocols. In most instances, this is likely to be adjusted dose heparin (70-100 international units/Kg) titrated to maintain an activated coagulation time (ACT) of $>300 \mathrm{~s}$. There is variability between catheterization laboratories regarding routine ACT assessment during PCI. We recommend that in cases with high burden of ICT, assessment of ACT may be useful to ensure that patients have had a therapeutic response to anticoagulation and may need additional heparin as required. Additionally, given the concern regarding efficacy of oral antiplatelet agents in pharmacologically naïve patients, a repeat ACT check at intervals and at procedure completion (with top-up heparin if required) may be prudent, although this strategy has not been proven in published trials to date.

Various ancillary pharmacological therapies have been utilized to treat distal embolization as a consequence of intracoronary thrombus formation. These agents include calcium channel blockers, adenosine, nicorandil, glycoprotein IIb/IIIa inhibitors, vasodilators and nitroprusside. Calcium channel blockers may inhibit platelet aggregation and have a direct effect on calcium flux through the sarcolemmal membrane that could protect injured myocytes [24, 25]. Adenosine affects intracellular calcium and inhibits neutrophil accumulation, superoxide generation $[26,27]$. Nicorandil, an ATP-dependent potassium channel opener, can prevent reperfusion injury and protect cardiac myocytes [28-30]. Glycoprotein IIB/IIIA inhibitors have also been used in cases of no reflow and inhibit platelet aggregation $[31,32]$. Each of these agents has variable success and none has been proven to be superior to another. Additionally, whether to administer these agents, pre- or poststent deployment is also undetermined. The clinical benefit of each of these individual agents on epicardial flow and myocardial salvage continues to be limited.

Intracoronary thrombolytic prior to aspiration thrombectomy could also be considered in selective cases. The DISSOLUTION trial compared intracoronary thrombolytic delivered via microcatheter and aspiration thrombectomy with aspiration 
thrombectomy alone. Patients treated with upfront thrombolytic had high rate of TIMI 3 flow and higher proportion of patients with myocardial blush grade of 2/3. Patients also had greater volume of aspirate compared to aspiration thrombectomy alone [33]. Further study is needed with larger clinical trials.

\subsection{Thrombectomy}

Although PPCI has been established as the dominant reperfusion strategy for the treatment of STEMI, the benefit of this approach can sometimes be limited. Mechanisms that may explain this limited benefit have included delays in time from symptom onset to reperfusion, reperfusion injury following vessel recanalization and distal embolization of thrombus. This lead to the development of thrombus removal with the concept that prevention of distal embolization might improve outcomes of primary PCI.

\subsubsection{Thrombectomy Systems}

Thrombectomy catheters while bulky confer benefits of better thrombus aspiration. Various systems exist that have different mechanisms of action which include rheolytic, fragmentation and manual thrombectomy. Rheolytic involves using highvelocity jets of saline which creates negative pressure through a Venturi effect. The AnjioJet is an example of a rheolytic system [34]. This device has lost its appeal after trials demonstrated an increased mortality with no difference in infarct size or resolution of ST segments $[35,36]$. Manual or 'aspiration' thrombectomy has been studied in large randomized controlled trials and emerged as the preferred method of thrombus removal primary due to its simplicity and ease of use.

Aspiration thrombectomy initially showed promise with benefit seen from early smaller randomized trials. These studies demonstrated the feasibility and potential benefit of thrombus extraction on indices of myocardial perfusion and ST segment resolution at time of PPCI. Infarct size was found to be less in patients undergoing thrombectomy before stenting compared with controls undergoing stenting without prior thrombectomy [37, 38]. These initial favourable studies lead to considerable uptake of routine manual thrombectomy during primary PCI. The advantages cited were improved reperfusion with less need for predilatation and arguably improved myocardial blush. Mechanistically, the postulated benefits of aspiration thrombectomy were less distal embolization and prevention of reperfusion injury. However, the large randomized TOTAL trial did not show any benefit for use of routine thrombectomy, and of concern there was a signal for harm with increased risk of stroke in the thrombectomy arm of the study [39-41]. The mechanism of stroke is likely due to aspiration back of clot and subsequent embolization to the brain.

In patient presenting with acute coronary syndrome and preexisting thrombus as a consequence of plaque rupture, routine thrombectomy is not recommended. While 
manual thrombectomy has a theoretical and intuitive basis for incremental value, results from pivotal trials and "real-world" registries of manual thrombectomy have not consistently demonstrated benefit over standard PCI [42]. Current European Society of Cardiology guidelines do not recommend routine use of thrombus aspiration. There may potentially be a benefit of selective thrombectomy. Patients who have very large thrombus may benefit, but this has not been robustly proven with randomized controlled evidence [43]. A recent meta-analysis of the three randomized trials showed a trend towards less cardiovascular death with aspiration thrombectomy compared to PCI. Of concern, there was also increased stroke or transient ischaemic attack despite a benefit in mortality. The pathophysiology is likely multifactorial and more complex, warranting further study. Additionally, thrombectomy may be helpful in cases where thrombus develops during PCI and after post-stent deployment. Aspiration thrombectomy may aid restoration of flow and help guide subsequent PCI.

\subsubsection{Suggested Technique for Manual Thrombectomy}

While routine aspiration thrombectomy is not advocated, there may be some patients that may benefit. In selected cases where a decision is made to proceed with thrombectomy, we suggest the following technique using the commonly available aspiration thrombectomy catheter.

- Ensure the guiding catheter is well engaged at all times.

- Start aspiration in the guiding catheter and make multiple passes until syringe is full.

- Continue to switch syringes as needed.

- Ensure a new syringe is placed with full negative pressure at time of removal of aspiration catheter. Prior to removal of the aspiration catheter, a small contrast test injection is advised to check that the guide is engaged.

- After removal of the aspiration catheter, we suggest allowing the guide catheter to bleed back or to aspirate through the side port of the manifold or autoinjector to ensure any clots that remain in the guide catheter are cleared prior to taking any further angiographic images.

Fastidious technique may reduce the likelihood of any subsequent stroke occurring.

\subsection{Other Causes of Intracoronary Thrombus Formation in STEMI Patients}

It is important to appreciate other causes of ICT that can occur in patients presenting with STEMI. While most patients present with ICT due to plaque rupture, patients can also present with STEMI and ICT due to embolism, vasospasm, spontaneous 
coronary artery dissection, coagulation disorders, trauma and endothelial dysfunction. In some cases, the underlying coronary artery is normal.

In cases where there is suspicion of another cause of STEMI other than plaque rupture, intracoronary imaging can be very helpful. In some cases, such as embolism, aspiration thrombectomy can aid restoration of vessel flow. Use of intravascular imaging with IVUS or OCT is very important to exclude underlying coronary artery disease. If the underlying vessel is normal, then stenting may not be required. In some causes such as spontaneous coronary artery dissection, diagnostic angiography and medical treatment only may be the optimal therapy for most cases. While intracoronary imaging can be used in cases of SCAD, routine use is not advocated as there is a risk of acute vessel closure with any further manipulation of the vessel.

\subsection{Stenting Strategy}

Large thrombus burden at time of primary PCI can lead to challenges with stent sizing, stent apposition and final TIMI flow, which can in turn increase the risk of stent thrombosis. While not recommended routinely, aspiration thrombectomy can be useful in cases of large thrombus burden to allow visualization of the vessel and appropriate stent sizing [43]. During PPCI judicious use of nitroglycerin is recommended to limit vasoconstriction which is common during STEMI due to inflammation and higher circulating catecholamines. Drug-eluting stents are recommended for PPCI. Some novel mesh-covered stents such as the MGuard stent which by design aim to prevent thrombus distal embolization have been trialled in small trials but have not been proven beneficial for routine use [44, 45].

\subsubsection{Deferred Stenting}

Primary PCI and stenting is the current recommended treatment for patients presenting with STEMI. This allows restoration of flow in the infarct artery and reduces reocclusion and restenosis. Implantation of a stent in a highly thrombotic milieu at the time of initial presentation with STEMI can be associated with risk of distal embolization and increased peri-procedural events. Furthermore, despite appropriate administration of antiplatelet agents, patients are often taken to the catheterization laboratory before these agents have achieved their desired therapeutic effect. One strategy to overcome this issue is 'deferred stenting', a practice that is not recommended routinely for all STEMI patients [46]. In selected cases, with high thrombus burden that are haemodynamically stable, deferred stenting may offer a therapeutic option. All patients should proceed urgently to the catheterization laboratory, and flow may need to be restored with a wire or gentle predilatation with a balloon. If high burden of thrombus is seen, deferred stenting with an interim period of adjunctive antiplatelet/anti thrombotic therapy may be useful to reduce the thrombus load and minimize complications (such as no-reflow) at time of stenting $[47,48]$. 


\subsection{Conclusion}

Intracoronary thrombus during STEMI is common and can lead to worse clinical outcomes. While there is no gold-standard therapy to deal with ICT, there are a combination of both pharmacological and mechanical therapies that can be utilized. Importantly, aspiration thrombectomy should not be used routinely in STEMI cases but may be helpful in selected cases at the discretion of the operator. The management of intracoronary thrombus at time of STEMI continues to remain a therapeutic challenge, but an awareness and aggressive management of ICT can lead to improved outcomes.

\subsection{Case Report: Heavy Thrombus Load in Primary PCI}

Janarthanan Sathananthan, Dale Murdoch, and Christopher Overgaard

\subsubsection{History}

A 42-year-old man with risk factors of type 2 diabetes and hypertension presented with a 6-h history of central chest pain and inferior ST segment elevation to a nonPCI capable hospital. He was loaded with aspirin $325 \mathrm{mg}$ and ticagrelor $180 \mathrm{mg}$ and transferred to a hospital capable of primary PCI. At time of presentation to the catheterization laboratory, he had ongoing chest discomfort and was bradycardic but haemodynamically stable.

\subsubsection{Management}

Arterial access was obtained via the transradial route. Intravenous heparin was administered at a dose of $100 \mathrm{IU} / \mathrm{kg}$. Diagnostic coronary angiography showed a critical occlusion in the right coronary artery with a significant ICT burden and TIMI 1 flow. Using a Judkins right guiding catheter, the right coronary artery was wired with a Balance Middle Weight coronary wire. Given the significant burden of ICT, a bolus dose and infusion of glycoprotein IIb/IIIa inhibitor were then administered. Following this, aspiration thrombectomy was performed to aid in restoration of vessel flow to prevent the need for balloon predilatation. Aspiration thrombectomy restored flow in the right coronary artery, but there was distal embolization of thrombus to the posterolateral vessel. Further aspiration thrombectomy was performed restoring flow and the culprit lesion was stented with a long drug-eluting stent. After stent deployment, there was evidence of no-reflow phenomenon. Repeated doses of intracoronary nitroglycerin and adenosine were administered. Subsequent angiographic pictures showed restoration of TIMI 3 flow and normal myocardial blush grade (Fig. 10.3). A left ventriculogram showed normal left ventricular function. 

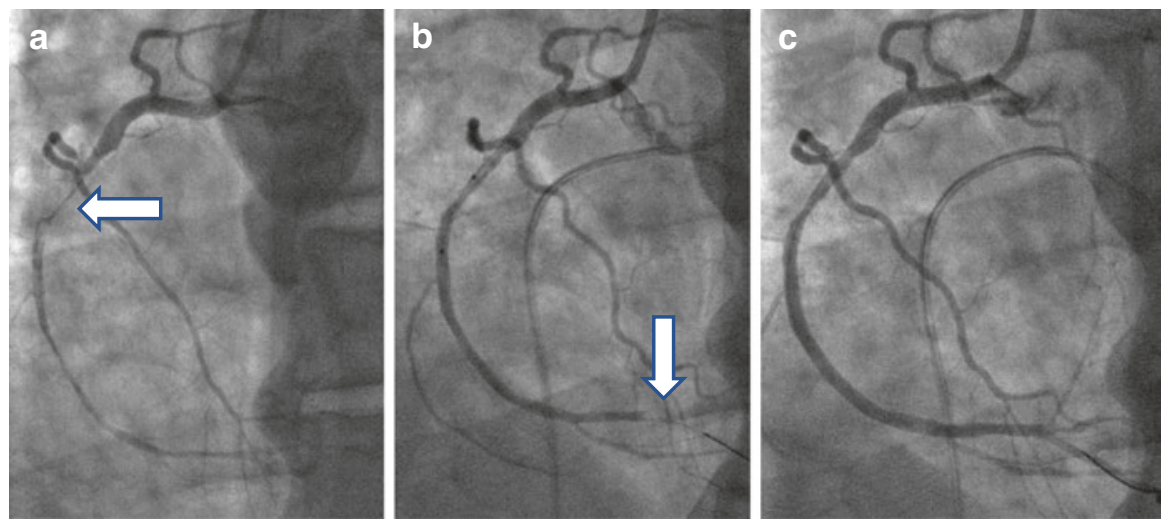

Fig. 10.3 Case example of intracoronary thrombus management during an inferior STEMI. (a) Initial diagnostic angiogram showing critical right coronary lesion with large thrombus (white arrow) at lesion. (b) Angiographic picture following aspiration thrombectomy with evidence of distal thrombus embolization to the posterolateral branch. (c) Angiographic picture following further thrombectomy and dose of glycoprotein IIb/IIIa inhibitor. (d) Final angiographic picture following stent deployment and doses of intracoronary adenosine and nitroglycerin to treat no reflow

\subsubsection{Outcome}

Following PPCI the patient's chest pain resolved. He had an uncomplicated recovery on the coronary care unit. Transthoracic echocardiography also showed normal left ventricular function. This case highlights that with judicious attention to the management of ICT, use of both pharmacological and percutaneous techniques can be used to successfully deal with ICT.

\subsection{Acute STEMI with Extensive Thrombus: An Illustrative Case}

Deborah Lee, Deanna Khoo, and Paul JL Ong

\subsubsection{Introduction}

Intracoronary thrombus poses a considerable challenge during percutaneous coronary intervention (PCI) and is associated with risk of no reflow, less intra-procedural success and increased in-hospital complications including myocardial infarction (MI) and death [49]. Many of these events are a direct result of distal embolization and microvascular obstruction. Consequently, various strategies to reduce thrombus burden have been explored. These include pharmacological thrombolysis, covered stents and thrombectomy - performed by manual aspiration or mechanical thrombus disruption. However, these strategies become a great challenge in the setting of large thrombus load. The use of stent retrievers for thrombus removal in acute 
ischaemic stroke has been shown to be safe and effective [39]. We describe the use of the Solitaire thrombus retrieval system (Medtronic MN, USA) in a patient with MI and heavy thrombus burden which was refractory to treatment by conventional means.

\subsubsection{Case Report}

A 54-year-old male smoker presented to the emergency department with a 2-day history of intermittent chest pain and anterior ST depression. He was loaded with aspirin $300 \mathrm{mg}$ and ticagrelor $180 \mathrm{mg}$ and referred for emergent cardiac catheterization.

Coronary angiography demonstrated the culprit to be an occluded distal left circumflex (LCx) artery (Fig. 10.4). During the procedure, he received enoxaparin and two boluses of intravenous eptifibatide. The occlusion was crossed easily with a Sion Blue wire (Asahi Intecc, JP) following which balloon angioplasty was performed with a $1.25 \mathrm{~mm}$ and then $2.0 \mathrm{~mm}$ compliant balloons inflated to nominal pressure. Although flow improved, a large intracoronary thrombus was now evident. Manual aspiration thrombectomy was performed with multiple runs of a $6 \mathrm{~F}$ Eliminate catheter (Terumo, JP) which only managed to retrieve small fragments of thrombus. As flow in the LCx had been restored, we opted for a strategy of deferred stenting $48 \mathrm{~h}$ later after completion of an eptifibatide infusion and ongoing therapeutic enoxaparin.

At repeat angiography, the thrombus burden was largely unchanged (Fig. 10.5). Having failed both aspiration thrombectomy and pharmacological thrombolysis, thrombectomy was attempted with a 2-4 $\mathrm{mm} \times 20 \mathrm{~mm}$ Solitaire thrombus retrieval

Fig. 10.4 Coronary angiogram of left coronary system. Note occlusion of the left circumflex artery

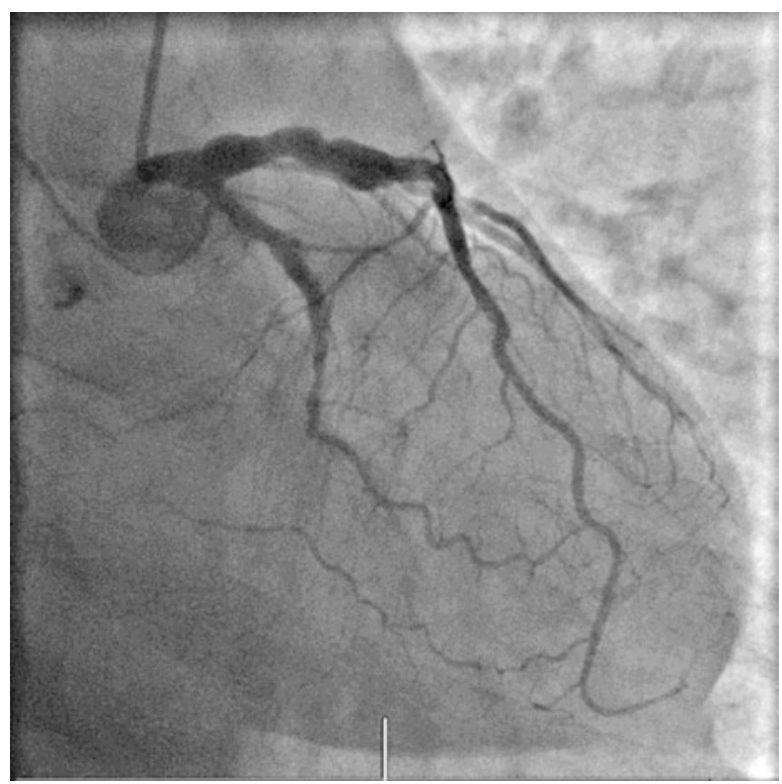


device. The left main was engaged with a 6F BL 3.5 guide catheter (Terumo), and a Sion Blue wire was advanced into the distal LCx, across the thrombus. A microcatheter was advanced through the wire and positioned distal to the thrombus. The selfexpanding Solitaire was then loaded inside the microcatheter and deployed across the thrombus (Fig. 10.6). The device was left expanded for $5 \mathrm{~min}$, following which it was carefully retrieved together with the microcatheter under continuous

Fig. 10.5 Coronary

angiogram after eptifibatide infusion. Note presence of large organized thrombus (arrow)

Fig. 10.6 Deployment of Solitaire device. Note the three dots visible at distal tip of Solitaire device. The microcatheter is still visible and will be used to partially collapse the proximal OD of the Solitaire and permit safe withdrawal
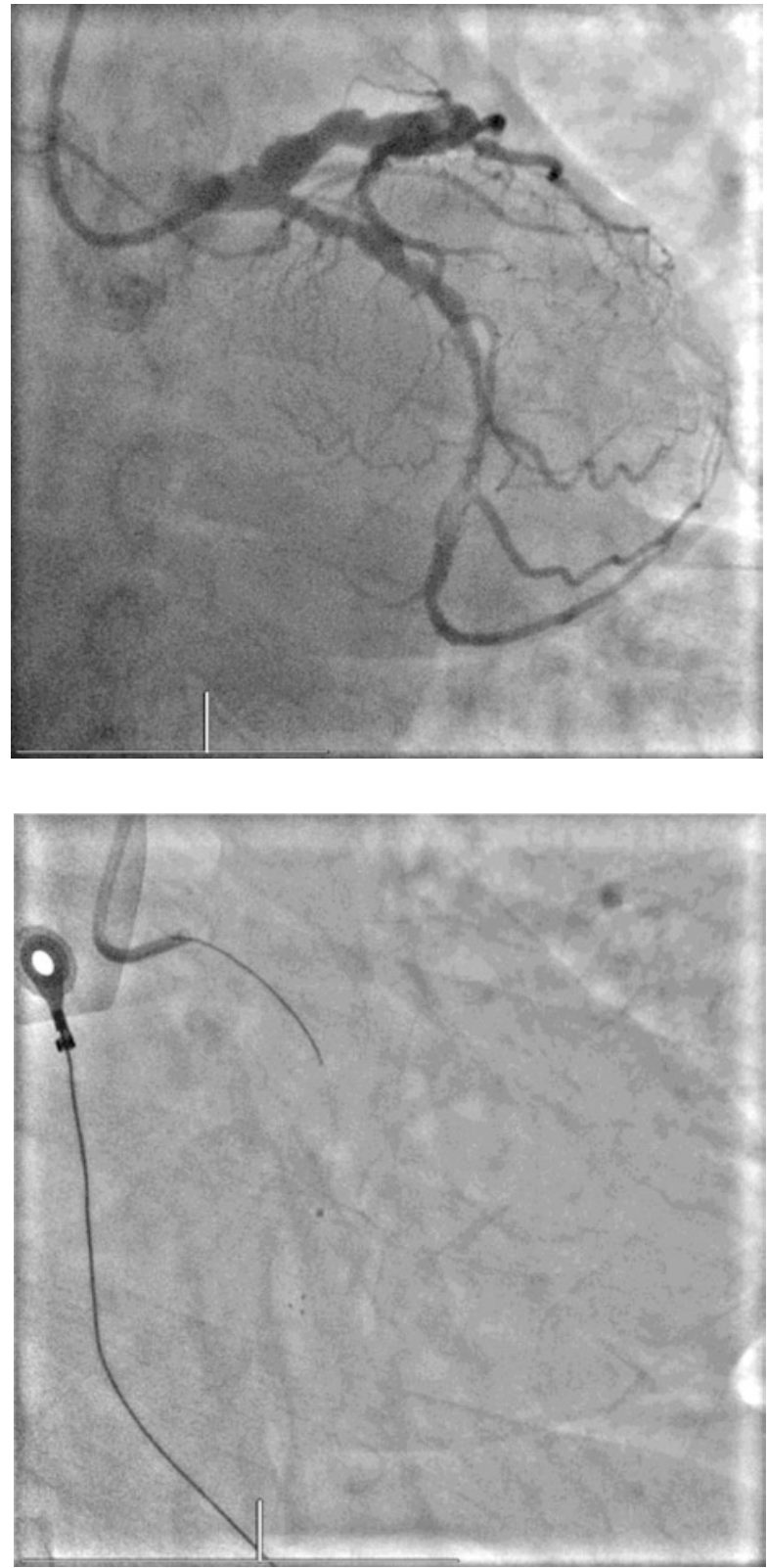
Fig. 10.7 Removal of Solitaire device. Note large thrombus entrapped within the struts of the device

Fig. 10.8 Coronary angiogram post thrombus retrieval. Note that the thrombus has largely been retrieved and the entirety of the circumflex artery is now widely patent
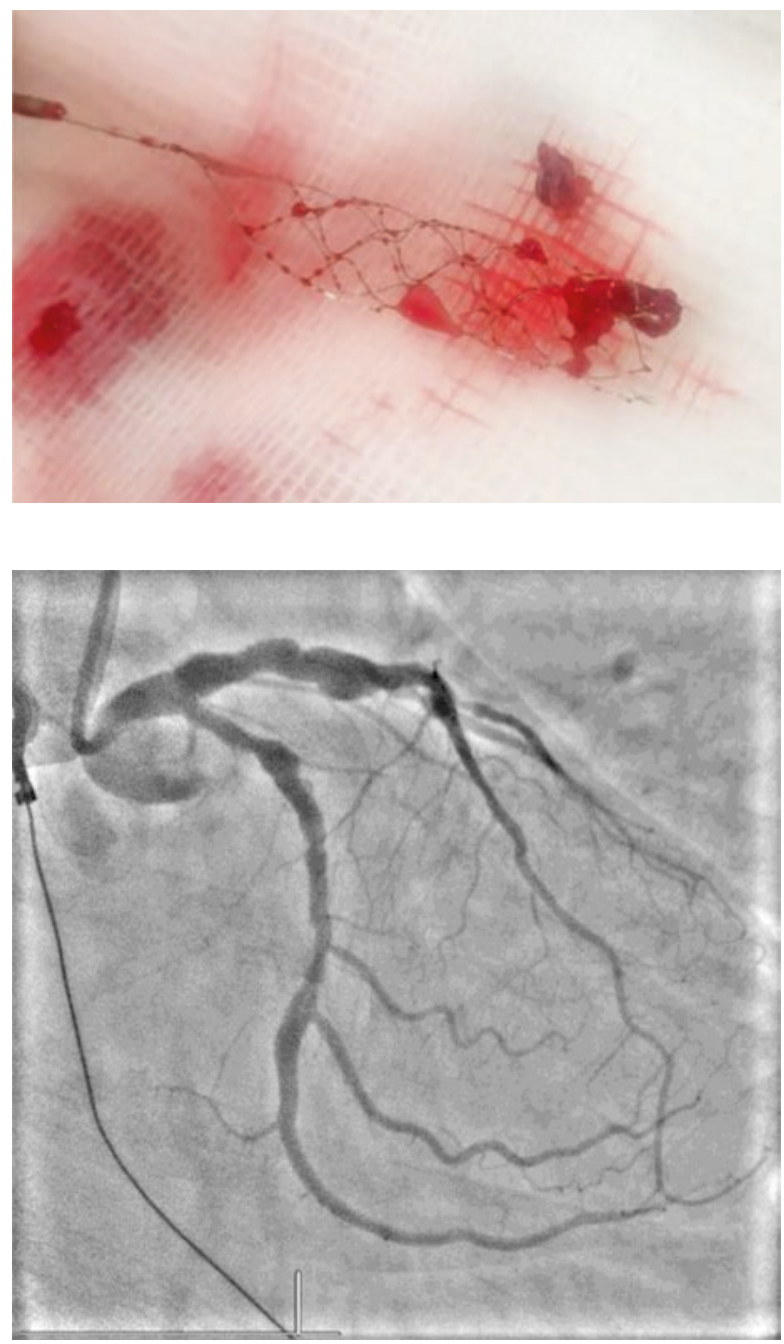

aspiration through the guide catheter. Device inspection showed successful retrieval of a large thrombus (Fig. 10.7). A TIMI three distal flow was accomplished (Fig. 10.8). The procedure was completed with a BioMatrix $3.5 \times 33 \mathrm{~mm}$ stent (Biosensor) being deployed across the culprit lesion. The patient was discharged stable 2 days later and has remained symptom-free ever since.

\subsubsection{Discussion}

Aspiration thrombectomy for ST-elevation MI is no longer considered routine due to no effect on clinical outcomes and association with stroke [39], thus in favour of a balloon strategy to restore coronary flow. However, in cases of persistent coronary 
thrombus - particularly when organized often leads to adverse sequelae and requires a tailored management approach.

In contrast to STEMI, manual thrombectomy in acute ischaemic stroke has been shown to be safe and is associated with improved recanalization rates and better functional outcomes [50]. Thrombus retrieval systems such as the Solitaire appear to be effective and are now class I guideline recommendation [51]. This device is easy to deliver even through tortuous cerebral vasculature; nonetheless reported complications include vessel dissection and thrombus embolization [52]. This case illustrates the potential use of this device to successfully retrieve the thrombus from the coronary circulation and further exploration of this strategy is warranted in a clinical trial.

\section{References}

1. Nabel EG, Braunwald E. A tale of coronary artery disease and myocardial infarction. N Engl J Med. 2012;366(1):54-63.

2. Furie B, Furie BC. Mechanisms of thrombus formation. N Engl J Med. 2008;359(9):938-49.

3. Davies MJ. The pathophysiology of acute coronary syndromes. Heart. 2000;83(3):361-6.

4. Falk E, Shah PK, Fuster V. Coronary plaque disruption. Circulation. 1995;92(3):657-71.

5. Topol EJ, Yadav JS. Recognition of the importance of embolization in atherosclerotic vascular disease. Circulation. 2000;101(5):570-80.

6. Gibson CM, de Lemos JA, Murphy SA, Marble SJ, McCabe CH, Cannon CP, et al. Combination therapy with abciximab reduces angiographically evident thrombus in acute myocardial infarction: a TIMI 14 substudy. Circulation. 2001;103(21):2550-4.

7. Sianos G, Papafaklis MI, Daemen J, Vaina S, van Mieghem CA, van Domburg RT, et al. Angiographic stent thrombosis after routine use of drug-eluting stents in ST-segment elevation myocardial infarction the importance of thrombus burden. J Am Coll Cardiol. 2007;50(7):573-83.

8. Niccoli G, Spaziani C, Marino M, Pontecorvo ML, Cosentino N, Bacà M, et al. Effect of chronic aspirin therapy on angiographic thrombotic burden in patients admitted for a first ST-elevation myocardial infarction. Am J Cardiol. 2010;105(5):587-91.

9. Fokkema ML, Vlaar PJ, Svilaas T, Vogelzang M, Amo D, Diercks GF, et al. Incidence and clinical consequences of distal embolization on the coronary angiogram after percutaneous coronary intervention for ST-elevation myocardial infarction. Eur Heart J. 2009;30(8):908-15.

10. Napodano M, Ramondo A, Tarantini G, Peluso D, Compagno S, Fraccaro C, et al. Predictors and time-related impact of distal embolization during primary angioplasty. Eur Heart $\mathrm{J}$. 2009;30(3):305-13.

11. Sharma V, Jolly SS, Hamid T, Sharma D, Chiha J, Chan W, et al. Myocardial blush and microvascular reperfusion following manual thrombectomy during percutaneous coronary intervention for ST elevation myocardial infarction: insights from the TOTAL trial. Eur Heart J. 2016;37(24):1891-8.

12. Stone GW, Peterson MA, Lansky AJ, Dangas G, Mehran R, Leon MB. Impact of normalized myocardial perfusion after successful angioplasty in acute myocardial infarction. J Am Coll Cardiol. 2002;39(4):591-7.

13. Patrono C. Aspirin as an antiplatelet drug. N Engl J Med. 1994;330(18):1287-94.

14. Wallentin L, Becker RC, Budaj A, Cannon CP, Emanuelsson H, Held C, et al. Ticagrelor versus clopidogrel in patients with acute coronary syndromes. N Engl J Med. 2009;361(11):1045-57.

15. Wessler JD, Stant J, Duru S, Rabbani L, Kirtane AJ. Updates to the ACCF/AHA and ESC STEMI and NSTEMI guidelines: putting guidelines into clinical practice. Am J Cardiol. 2015;115(5 Suppl):23A-8A. 
16. Montalescot G, van 't Hof AW. Prehospital ticagrelor in ST-segment elevation myocardial infarction. N Engl J Med. 2339;371(24):2014.

17. Ernst NM, Suryapranata H, Miedema K, Slingerland RJ, Ottervanger JP, Hoorntje JC, et al. Achieved platelet aggregation inhibition after different antiplatelet regimens during percutaneous coronary intervention for ST-segment elevation myocardial infarction. J Am Coll Cardiol. 2004;44(6):1187-93.

18. Bhatt DL, Stone GW, Mahaffey KW, Gibson CM, Steg PG, Hamm CW, et al. Effect of platelet inhibition with cangrelor during PCI on ischemic events. N Engl J Med. 2013;368(14):1303-13.

19. Stone GW, Mehran R, Goldstein P, Witzenbichler B, Van 't Hof A, Guagliumi G, et al. Bivalirudin versus heparin with or without glycoprotein IIb/IIIa inhibitors in patients with STEMI undergoing primary percutaneous coronary intervention pooled patient-level analysis from the HORIZONS-AMI and EUROMAX trials. J Am Coll Cardiol. 2015;65(1):27-38.

20. Yusuf S, Mehta SR, Chrolavicius S, Afzal R, Pogue J, Granger CB, et al. Effects of fondaparinux on mortality and reinfarction in patients with acute ST-segment elevation myocardial infarction: the OASIS-6 randomized trial. JAMA. 2006;295(13):1519-30.

21. Routledge H, Sastry S. Radial versus femoral access for acute coronary syndromes. Curr Cardiol Rep. 2015;17(12):117.

22. Jolly SS, Yusuf S, Cairns J, Niemelä K, Xavier D, Widimsky P, et al. Radial versus femoral access for coronary angiography and intervention in patients with acute coronary syndromes (RIVAL): a randomised, parallel group, multicentre trial. Lancet. 2011;377(9775):1409-20.

23. Shahzad A, Kemp I, Mars C, Wilson K, Roome C, Cooper R, et al. Unfractionated heparin versus bivalirudin in primary percutaneous coronary intervention (HEAT-PPCI): an open-label, single Centre, randomised controlled trial. Lancet. 2014;384(9957):1849-58.

24. Pomerantz RM, Kuntz RE, Diver DJ, Safian RD, Baim DS. Intracoronary verapamil for the treatment of distal microvascular coronary artery spasm following PTCA. Catheter Cardiovasc Diagn. 1991;24(4):283-5.

25. Kaplan BM, Benzuly KH, Kinn JW, Bowers TR, Tilli FV, Grines CL, et al. Treatment of noreflow in degenerated saphenous vein graft interventions: comparison of intracoronary verapamil and nitroglycerin. Catheter Cardiovasc Diagn. 1996;39(2):113-8.

26. Micari A, Belcik TA, Balcells EA, Powers E, Wei K, Kaul S, et al. Improvement in microvascular reflow and reduction of infarct size with adenosine in patients undergoing primary coronary stenting. Am J Cardiol. 2005;96(10):1410-5.

27. Movahed MR, Baweja G. Distal administration of very high doses of intracoronary adenosine for the treatment of resistant no-reflow. Exp Clin Cardiol. 2008;13(3):141-3.

28. Sakata Y, Kodama K, Ishikura F, Komamura K, Hasegawa S, Hirayama A. Disappearance of the 'no-reflow' phenomenon after adjunctive intracoronary administration of nicorandil in a patient with acute myocardial infarction. Jpn Circ J. 1997;61(5):455-8.

29. Matsuo H, Watanabe S, Watanabe T, Warita S, Kojima T, Hirose T, et al. Prevention of noreflow/slow-flow phenomenon during rotational atherectomy-a prospective randomized study comparing intracoronary continuous infusion of verapamil and nicorandil. Am Heart J. 2007;154(5):994.e1-6.

30. Ito H, Taniyama Y, Iwakura K, Nishikawa N, Masuyama T, Kuzuya T, et al. Intravenous nicorandil can preserve microvascular integrity and myocardial viability in patients with reperfused anterior wall myocardial infarction. J Am Coll Cardiol. 1999;33(3):654-60.

31. de Lemos JA, Antman EM, Gibson CM, McCabe CH, Giugliano RP, Murphy SA, et al. Abciximab improves both epicardial flow and myocardial reperfusion in ST-elevation myocardial infarction. Observations from the TIMI 14 trial. Circulation. 2000;101(3):239-43.

32. Neumann FJ, Blasini R, Schmitt C, Alt E, Dirschinger J, Gawaz M, et al. Effect of glycoprotein IIb/IIIa receptor blockade on recovery of coronary flow and left ventricular function after the placement of coronary-artery stents in acute myocardial infarction. Circulation. 1998;98(24):2695-701.

33. Greco C, Pelliccia F, Tanzilli G, Tinti MD, Salenzi P, Cicerchia C, et al. Usefulness of local delivery of thrombolytics before thrombectomy in patients with ST-segment elevation myocardial infarction undergoing primary percutaneous coronary intervention (the delivery of throm- 
bolytics before thrombectomy in patients with ST-segment elevation myocardial infarction undergoing primary percutaneous coronary intervention [DISSOLUTION] randomized trial). Am J Cardiol. 2013;112(5):630-5.

34. Migliorini A, Stabile A, Rodriguez AE, Gandolfo C, Rodriguez Granillo AM, Valenti R, et al. Comparison of AngioJet rheolytic thrombectomy before direct infarct artery stenting with direct stenting alone in patients with acute myocardial infarction. The JETSTENT trial. J Am Coll Cardiol. 2010;56(16):1298-306.

35. Ali A, Cox D, Dib N, Brodie B, Berman D, Gupta N, et al. Rheolytic thrombectomy with percutaneous coronary intervention for infarct size reduction in acute myocardial infarction: 30-day results from a multicenter randomized study. J Am Coll Cardiol. 2006;48(2):244-52.

36. Parodi G, Valenti R, Migliorini A, Maehara A, Vergara R, Carrabba N, et al. Comparison of manual thrombus aspiration with rheolytic thrombectomy in acute myocardial infarction. Circ Cardiovasc Interv. 2013;6(3):224-30.

37. Svilaas T, Vlaar PJ, van der Horst IC, Diercks GF, de Smet BJ, van den Heuvel AF, et al. Thrombus aspiration during primary percutaneous coronary intervention. $\mathrm{N}$ Engl J Med. 2008;358(6):557-67.

38. Lagerqvist B, Fröbert O, Olivecrona GK, Gudnason T, Maeng M, Alström P, et al. Outcomes 1 year after thrombus aspiration for myocardial infarction. $\mathrm{N}$ Engl $\mathrm{J}$ Med. 2014;371(12):1111-20.

39. Jolly SS, Cairns JA, Yusuf S, Meeks B, Pogue J, Rokoss MJ, et al. Randomized trial of primary PCI with or without routine manual thrombectomy. N Engl J Med. 2015;372(15):1389-98.

40. Jolly SS, Cairns JA, Yusuf S, Rokoss MJ, Gao P, Meeks B, et al. Outcomes after thrombus aspiration for ST elevation myocardial infarction: 1-year follow-up of the prospective randomised TOTAL trial. Lancet. 2016;387(10014):127-35.

41. Bhindi R, Kajander OA, Jolly SS, Kassam S, Lavi S, Niemelä K, et al. Culprit lesion thrombus burden after manual thrombectomy or percutaneous coronary intervention-alone in ST-segment elevation myocardial infarction: the optical coherence tomography sub-study of the TOTAL (ThrOmbecTomy versus PCI ALone) trial. Eur Heart J. 2015;36(29):1892-900.

42. Jolly SS, James S, Džavík V, Cairns JA, Mahmoud KD, Zijlstra F, et al. Thrombus aspiration in ST-segment-elevation myocardial infarction: an individual patient meta-analysis: Thrombectomy Trialists collaboration. Circulation. 2017;135(2):143-52.

43. Ibanez B, James S, Agewall S, Antunes MJ, Bucciarelli-Ducci C, Bueno H, et al. 2017 ESC guidelines for the management of acute myocardial infarction in patients presenting with ST-segment elevation: The Task Force for the management of acute myocardial infarction in patients presenting with ST-segment elevation of the European Society of Cardiology (ESC). Eur Heart J. 2017;39(2):119-77.

44. Stone GW, Abizaid A, Silber S, Dizon JM, Merkely B, Costa RA, et al. Prospective, randomized, multicenter evaluation of a polyethylene terephthalate micronet mesh-covered stent (MGuard) in ST-segment elevation myocardial infarction: the MASTER trial. J Am Coll Cardiol. 2012;60(19):1975-84.

45. Gracida M, Romaguera R, Jacobi F, Gómez-Hospital JA, Cequier A. The MGuard coronary stent: safety, efficacy, and clinical utility. Vasc Health Risk Manag. 2015;11:533-9.

46. Qiao J, Pan L, Zhang B, Wang J, Zhao Y, Yang R, et al. Deferred versus immediate stenting in patients with ST-segment elevation myocardial infarction: a systematic review and metaanalysis. J Am Heart Assoc. 2017;6(3):pii: e004838.

47. De Maria GL, Alkhalil M, Oikonomou EK, Wolfrum M, Choudhury RP, Banning AP. Role of deferred stenting in patients with ST elevation myocardial infarction treated with primary percutaneous coronary intervention: a systematic review and meta-analysis. J Interv Cardiol. 2017;30(3):264-73.

48. Carrick D, Oldroyd KG, McEntegart M, Haig C, Petrie MC, Eteiba H, et al. A randomized trial of deferred stenting versus immediate stenting to prevent no- or slow-reflow in acute ST-segment elevation myocardial infarction (DEFER-STEMI). J Am Coll Cardiol. 2014;63(20):2088-98. 
49. Singh M, Berger PB, Ting HH, et al. Influence of coronary thrombus on outcome of percutaneous coronary angioplasty in the current era (the Mayo Clinic experience). Am J Cardiol. 2001;88:1091-6.

50. Davalos A, Pereira VM, Chapot R. Retrospective multicenter study of solitaire FR for revascularization in the treatment of acute ischemic stroke. Stroke. 2012;43:2699-705.

51. Jauch EC, Saver JL, et al. Guidelines for the early Management of Patients with Acute Ischemic Stroke. Stroke. 2013;44:870-947.

52. Elgendy IY, Kumbhani DJ, et al. Mechanical thrombectomy for acute ischaemic stroke: a meta-analysis of randomized trials. JACC. 2015;66:2498-505.

Open Access This chapter is licensed under the terms of the Creative Commons Attribution 4.0 International License (http://creativecommons.org/licenses/by/4.0/), which permits use, sharing, adaptation, distribution and reproduction in any medium or format, as long as you give appropriate credit to the original author(s) and the source, provide a link to the Creative Commons license and indicate if changes were made.

The images or other third party material in this chapter are included in the chapter's Creative Commons license, unless indicated otherwise in a credit line to the material. If material is not included in the chapter's Creative Commons license and your intended use is not permitted by statutory regulation or exceeds the permitted use, you will need to obtain permission directly from the copyright holder. 